\title{
Essai d'affaissement d'un blindage cylindrique de galerie muni de renforts et soumis à la pression extérieure
}

\author{
Collapsing test \\ on stiffened cylindrical tunnel sheeting \\ subjected to an outside pressure
}

\author{
PAR J. RIGAL \\ INGÉNIEUR DES ARTS ET MÉTIERS \\ ÉTABLISSEMENTS BOUGHAYER \& VIALLET, GRENOBLE
}

\begin{abstract}
Cet essai, demandé par Electricité de France, à l'occasion de l'exécution des galeries blindées de la chute de Roselend, a été effectué sur un tuyau de $3800 \mathrm{~mm}$ de diamètre, de $12 \mathrm{~mm}$ d'épaisseur et de $2700 \mathrm{~mm}$ de longueur.

$1 l$ avait pour but de vérifier le comportement d'un tel tuyau muni de renforts extérieurs en cornières, soumis da la pression d'eau interstitielle contenue dans la roche. Des essais analogues avaient été précédemment faits, mais sur un tuyau non muni de renforts.

Après un exposé, critique des diverses théories actuellement connues concernant l'affaissement des enveloppes cylindriques, en particulier de celles munies de renforts annulaires, l'auteur expose la conception de l'essai, décrit le modèle, les appareils de mesure, le déroulement des essais et leur résultat final.

L'étude se termine par une description critique sur les précisions dur calcul et sur les résultats obtenus.
\end{abstract}

\begin{abstract}
This test was asked for by Electricité de France when the tunnel sheeting was being manufactured for the Roselend project, and was carried out on a pipe with a diameter of $3800 \mathrm{mms}$, a length of $2700 \mathrm{mms}$ and $12 \mathrm{mms}$ walls.

The purpose of the test was to find out how the sheeting, which was fitted with external angle iron stiffeners, would stand up to the pressure of the interstitial water in the surrounding rock. Similar tests had previonsly- been undertaken with unstiffened sheeting.

A critical discussion of various current theories on the collapsing of cylindrical shells-especially when fitted with annular stiffeners-is followed by a description of the test particulars, the model, its instrumentation, the actual tests, and their final result. The article ends with a critical assessment of the calculation accuracy and the results obtained.
\end{abstract}

\section{INTRODUCTION}

La résistance aux sous-pressions des blindages cylindriques en galeries bétonnées a fait l'objet de nombreuses études, et les conceptions des constructeurs diffèrent sur les renforcements à utiliser et sur leur justification.

L'étude présentée a été effectuée pour Electricité de France qui avait confié aux Etablissements Bouchayer et Viallet l'exécution des blindages des galeries d'amenée à la conduite forcée de Roselend, pour lesquelles les pressions extérieures étaient supérieures aux pressions internes.
Les caractéristiques des blindages des galeries d'amenée de Roselend sont :

- Diamètre du blindage $3800 \mathrm{~mm}$;

- Epaisseur des viroles $12 \mathrm{~mm}$;

- Cercles renfort en cornières $120 \times 120 \times 13$ posées en chevron et soudées, espacées d'axe en axe de $675 \mathrm{~mm}$;

- Pression intérieure $16,5 \mathrm{~kg} / \mathrm{cm}^{2}$;

- Pression extérieure $20 \mathrm{~kg} / \mathrm{cm}^{2}$.

Acier employé :

- Acier A $55 \mathrm{M}$ avec $\mathrm{R} \geqslant 54 \mathrm{~kg} / \mathrm{mm}^{2}$; 
$-\mathrm{E} \geqslant 36 \mathrm{~kg} / \mathrm{mm}^{2} \quad \mathrm{~A} \geqslant 20 \%$;

- Cornière renfort de même qualité.

Les résultats des essais ont démontré la valeur de la théorie de l'affaissement des blindages lis- ses, établie par M. Simon-Suisse, et ils ont justifié la continuation des études dans le même sens pour son application aux blindages renforcés par anneaux.

\section{I. - BUT ET RAISONS DE L'ESSAI}

L'avant-projet de blindage de la galerie d'amenée de Roselend avait conduit Electricité de France, R.E.H. - Alpes II, et les Etablissements Bouchayer et Viallet à prévoir la constitution de ce blindage en partant d'une tôle mince soutenue à faibles intervalles par des raidisseurs formés de cornières placées «en chevrons». L'intervalle entre le blindage et le rocher était prévu rempli de béton, selon le principe habituel.

L'étude comparative des prix de revient de ce système et du système classique de tôles lisses, établie dans les mêmes conditions, avec les mêmes charges extérieures, en tolérant les mêmes contraintes et rapports 《de sécurité », en utilisant des modes de calculs similaires et des aciers identiques, faisait apparaître l'avantage économique de la solution tôle mince et raidisseurs par rapport à la seconde.

Le système prévu était donc celui représenté sur la figure 1.

\section{***}

Cependant, l'utilité d'une confirmation expérimentale des dimensionnements semblait nécessaire. En effet :

- Toutes les théories connues à l'époque sur l'affaissement des blindages en galerie étaient

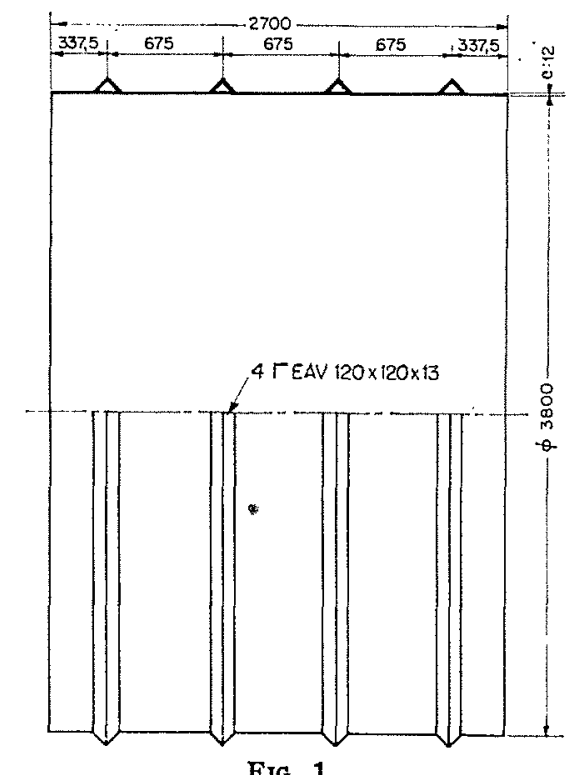

FIG. 1

Virole Roselend. mathématiquement mal fondées, leur foisonnement n'en étant que plus inquiétant. D'autre part, le peu d'informations recueillies sur les cas d'incidents qui se sont produits sur les installations existantes, n'a pas permis d'en tirer les enseignements pratiques souhaitables.

La détermination des renforts avait nécessité l'extension d'une des meilleures formules, celle de E. Amstütz, établie initialement pour des blindages lisses. C'est d'ailleurs cette extension qui nous avait révélé les faiblesses mathématiques de cette théorie.

- Par ailleurs la région d'équipement hydraulique Alpes I, effectuait en commun avec un constructeur, un essai similaire sur des viroles de dimensions comparables. L'appareillage à mettre en jeu pouvait donc être partiellement réutilisé.

- Enfin l'aménagement de Roselend, par son importance et par le tonnage mis en jeu pour la construction du seul blindage, justifiait l'exécution de cet essai.

$$
\text { 森 }
$$

Il fut donc décidé d'effectuer des essais, sur une première virole, réalisée à l'échelle 1 (une similitude étant impossible à établir tant qu'il n'existe pas une théorie indiscutable); selon les résultats obtenus, d'autres viroles pourraient être mises en essai. Les buts étaient les suivants :

$1^{\circ}$ S'assurer, indépendamment de toute vérification de note de calculs, que la virole était effectivement capable de supporter les pressions prévues en service;

$2^{\circ}$ Comparer les résultats d'essai avec les prévisions de la note de calculs;

$3^{\circ}$ Confronter - dans toute la mesure du possible - les observations faites au cours de l'essai et ses résultats avec toute théorie connue. $\mathrm{Au}$ besoin se servir de ces résultats pour orienter la recherche d'une théorie valable.

Ces deux derniers points présentaient plus spécialement la part de travail des Etablissements Bouchayer et Viallet.

\section{秝}

L'essai ayant montré - comme nous le verrons plus loin - que la virole satisfaisait au premier point, les deux points suivants deviennent les plus importants, et c'est donc sur eux que portera cette communication. 


\section{II. - THEORIES AYANT SERVI DE BASES A L'ETUDE DU BLINDAGE ET A L'INTERPRETATION DE L'ESSAI}

\section{II.1 - Rappel théorique sur le flambage.}

Les études sur le flambage classique font apparaître deux stades :

- Flambage d'un système homogène et géométriquement parfait;

-.- Flambage d'un système non homogène, ou géométriquement imparfait, ou soumis à des forces latérales.

Pour résumer très succinctement ce problème, on peut dire que, lors du premier stade d'étude, on fait apparaître qu'un système géométriquement parfait et homogène, soumis à une force de membrane se déforme en restant semblable à Iui-même, jusqu'à ce que la force de membrane atteigne une valeur de compression dite force critique, d'ordre $1,2,3, \ldots n$, selon qu'elle est, dans l'ordre des compressions croissantes, la première, la seconde, la troisième ou la nième. Nommons ces forces F1, F2, F3 ... Fn. Jusqu'au dernier moment, le système était resté semblable à lui-même; il se déforme alors brutalement d'une manière non. semblable. Cette déformation peut être représentée par :

$$
z=a_{n} f_{n}(x, y)
$$

$f_{n}$ est une fonction parfaitement déterminée, correspondant à la force critique d'ordre $n$, tandis que le coefficient $a_{n}$, est lui, rigoureusement indéterminé et peut devenir infini.

Les fonctions $f_{n}$, généralement d'allure sinusoïdale (l'équation différentielle étant d'ordre 2), sont appelées «mode $n$ », par abréviation de mode de déformation d'ordre $n$. Ces fonction sont toutes orthogonales entre elles, c'est-à-dire que :

$$
\iint_{s} f_{n}(x, y) \times f_{p}(x, y) d x . d y=0
$$

lorsque l'intégrale est étendue à l'ensemble du système.

On a pu constater qu'un système réel ne se comporte pas de cette manière. En réalité, dès l'apparition de la moindre force de compression, des déformations non en similitude, aussi faibles soient-elles, apparaissent. C'est là qu'intervient le second stade.

Le cas présenté, qui est le plus simple et le plus fréquent, est celui du système géométriquement imparfait, et non celui du système non homogène ou soumis à des charges latérales. Soit $z_{0}=g_{0}(x, y)$, la fonction représentant ses imperfections initiales par rapport à la forme de membrane. Les différents modes étant orthogonaux entre eux et répondant aux conditions aux extrémités, la fonction $g(x, y)$ peut être représentée par une série des divers modes (analogues à la série de Fourier).

Soit cette série :

$$
\begin{aligned}
z_{0}=g_{0}(x, y)=a_{0} & +a_{1} f_{1}(x, y) \\
& +a_{2} f_{2}(x, y)+\ldots+a_{n} f_{n}(x, y)
\end{aligned}
$$

Lorsque la force de membrane $F$ quelconque est appliquée, on démontre que la déformation devient :

$$
\begin{aligned}
z_{\mathrm{F}}=g_{\mathrm{F}}(x, y)= & \alpha_{0}^{\prime}+a_{1}^{\prime} f_{1}(x, y) \\
& +a^{\prime}{ }_{2} f_{2}(x, y)+\ldots+\alpha_{n}^{\prime} f_{n}(x, y)
\end{aligned}
$$

où les valeurs $a$ et $a^{\prime}$ sont liées par la relation :

$$
a_{i}^{\prime}=a_{i} \times \frac{1}{1-\left(\mathrm{F} / \mathrm{F}_{i}\right)}
$$

On voit immédiatement que $a_{i}^{\prime}$ prend une valeur hyperbolique en fonction de $F$, et que $F_{i}$ représente un pôle.

L'existence de pôles, et le rôle fondamental de l'allure des déformations initiales dans l'état de déformations final est la caractéristique fondamentale des phénomènes de flambage.

\section{II.2 - Théories existantes de l'affaissement d'un blindage en galerie lors du projet de Roselend.}

\section{II.2.1. - AFFAISSEMENT DU RAIDISSEUR :}

Lors de l'établissement du projet de Roselend, les théories alors connues de l'affaissement d'un blindage en galerie se rapportaient toutes à des blindages lisses, et la plus connue était celle de E. Amstütz.

La formule est basée sur la théorie suivante : au cours de l'établissement de la pression extérieure, il apparaît une cloque, dont la déforméc répond aux équations différentielles de l'équilibre. Par ailleurs, l'amplitude de cette cloque est telle que le développement de la figure déformée soit égal à la longueur initiale du système diminuée des raccourcissements dus à l'effort de membrane. Ces deux hypothèses permettent de poser deux équations dont la résolution donne l'amplitude maximale de la déformée en fonction de la pression. Cette amplitude connue, on peut calculer la contrainte en tout point, et E. Amstütz admet que lorsque la contrainte atteint la limite ćlastique, les déformations sont si importantes que la ruine est pratiquement atteinte. Il calcule donc la pression, ou la contrainte moyenne de 
membrane pour laquelle la limite élastique est atteinte. Cette contrainte moyenne $\sigma_{c r}$, peut être calculée à l'aide de la formule suivante :

$$
\begin{aligned}
& \left(\frac{\varepsilon}{\mathrm{R}}+\frac{\sigma_{c r}}{\mathrm{E}+}\right) \sqrt{\left(1+12 \frac{\mathrm{R}^{2}}{e^{2}} \frac{\sigma_{c r}}{\mathrm{E}+}\right)^{3}} \\
& \quad=3,36 \frac{\mathrm{R}}{e} \frac{\sigma_{\mathrm{E}}-\sigma_{c r}}{\mathrm{E}+}\left(1-0,5 \frac{\mathrm{R}}{e} \frac{\sigma_{\mathrm{E}}-\sigma_{c r}}{\mathrm{E}+}\right)
\end{aligned}
$$

équation dans laquelle

$\varepsilon=$ jeu radial entre virole et béton;

$\mathrm{R}=$ rayon de la virole;

$e=$ épaisseur de la virole;

$\mathrm{E}+=\frac{\mathrm{E}_{(\text {module d'elasticité) }}}{1-v^{2}}$

$\sigma_{\mathrm{E}}=$ limite élastique du métal utilisé.

Cette formule est de résolution graphique relativement aisée.

L'extension de cette formule au cas d'un renfort est assez simple. En suivant exactement le même développement, on établit la formule :

$$
\begin{aligned}
& \left(\frac{\varepsilon}{\mathrm{R}}+\frac{\sigma_{c r}}{\mathrm{E}+}\right) \sqrt{\left(1+\frac{\mathrm{R}^{2}}{\rho^{2}} \frac{\sigma_{c r}}{\mathrm{E}+}\right)^{3}} \\
& \quad=1,68 \frac{\mathrm{R}}{v} \frac{\sigma_{\mathrm{E}}-\sigma_{c r}}{\mathrm{E}+}\left(1-0,25 \frac{\mathrm{R}}{v} \frac{\sigma_{\mathrm{E}}-\sigma_{c r}}{\mathrm{E}+}\right)
\end{aligned}
$$

dans laquelle $\sigma_{c r}$ est la contrainte moyenne (établie selon la théorie des coques de révolution) provoquant l'affaissement du raidisseur, $\rho$, le rayon de giration de la section raidisseur + tôle associée, $v$, la distance du point le plus éloigné à l'axe neutre, les autres symboles conservant leur signification précédente.

Dans la mesure où il était possible de connâ̂tre la largeur de tôle associée au raidisseur, cette formule pour autant qu'on l'accepte, permettait de résoudre le problème du raidisseur.

Or, une étude de Timoshenko (théorie de l'élasticité), montre que dans le cas particulier d'une charge sinusoïdale de période $l$, la largeur efficace est indépendante des caractéristiques géométriques de la section, et vaut $0,18 \mathrm{l}$ environ. Le facteur dominant de cette largeur associée reste d'ailleurs dans tous les cas la longueur de la période.

Comme par ailleurs la théorie de E. Amstütz admet une déformée sinusoïdale, et que plusieurs études faites par les Etablissements Bouchayer et Viallet sur cette théorie avaient montré que, dans la majorité des cas courants, la période de la cloque était proche de $\pi \mathrm{R} / 2$ (soit $\left.90^{\circ}\right)$, il a été admis pour largeur associée :

$$
\frac{\pi \mathrm{R}}{2} \times 0,18 \cong 0,285 \mathrm{R}
$$

A priori, la théorie de E. Amstütz semble en bon accord avec les données physiques.

Cependant l'étude détaillée entreprise lors de l'extension au cas du raidisseur, avait fait apparaître une erreur de principe dans la mise en équation $d u$ problème : l'équation différentielle choisie par E. Amstütz (équation linéaire du second ordre à coefficients constants) ne permet pas de satisfaire aux conditions d'extrémités. E. Amstütz a d'ailleurs présenté successivement deux formules. Dans la première, où il choisissait une déformée comportant une période sinusoïdale, apparaissait une discontinuité de moments aux points de contacts sur le béton. Dans la seconde, où il choisit une déformée à trois demi-périodes, la discontinuité de moments disparaît, mais est remplacée par une discontinuité d'efforts tranchants. Cette discontinuité est absorbée par les réactions d'appui aux points de contact avec le béton, mais si l'on tient compte de ces réactions d'appui, les équations différentielles de l'équilibre ne sont plus linéaires. Comme indiqué plus loin, la solution exacte du problème a été donnée peu de temps avant que n'ait lieu l'essai, et uniquement dans le cas d'une tôle lisse.

\section{II.2.2. - CloquAGE DE LA PAROI ENTRE RAIDIS-} SEURS :

Hors de l'affaissement du raidisseur - qui doit être considéré comme l'incident majeur peut intervenir un incident mineur, qui est le cloquage de la peau du blindage. Le flambage d'une paroi cylindrique entre deux raidisseurs annulaires, infiniment rigides, est un phénomène bien connu, et parfaitement prévisible. On peut en trouver la théorie complète dans Timoshenko : "Théorie de la stabilité élastique», et dans R. G. Sturm «A study of collapsing pressure of thin walled cylinders $\gg$.

L'existence d'un jeu entre la paroi et le béton justifiait l'utilisation de la même théorie pour l'étude de ce phénomène. En effet, si l'existence du béton pouvait gêner la fin de ce phénomène, elle ne pouvait pas influer sur le début, en raison précisément du jeu.

\section{II.3 - Théorie de l'affaissement du blindage lisse.}

Peu de temps avant l'exécution de l'essai, M. Simon-Suisse, alors Directeur des Etudes aux Etablissements Bouchayer et Viallet, établissait une théorie de l'affaissement des blindages lisses de galeries.

Cette théorie est basée sur des hypothèses identiques à celles de E. Amstütz, à savoir : appui continu sur une partie du béton, et décollement sur une zone et une seule, formant cloque, cette cloque devenant de plus en plus profonde avec 
l'augmentation de la pression, jusqu'à ce que les contraintes dépassent la limite élastique.

Par contre, la résolution des équations différentielles et des équations de l'équilibre y est traitée avec une rigueur mathématique incontestable. La solution — pour la déformée - y apparaît sous la forme d'une fonction de Mathieu. La théorie montre comment dans la majorité des cas pratiques, dont on détermine les limites, la résolution de l'équation de Mathieu peut être simplifiée et elle aboutit à un abaque d'emploi très simple.

Il semble cependant qu'une seule question pouvait être posée concernant cette théorie : si les frottements sur le béton avaient une influence relativement faible, il devait effectivement se former une seule cloque, ce cas étant celui qui correspond à la pression d'affaissement la plus basse. Par contre, si les frottements étaient importants, la déformation en 8 qui marque le début du phénomène provoquerait l'appui en deux points diamètralement opposés, puis la formation de deux cloques persistantes.

On doit remarquer que dans le développement de la théorie n'apparaît aucune valeur polaire similaire à celles que sont les pressions critiques. Pour une pression, il existe une déformẻe et une seule, indépendante de l'amplitude des déformations initiales. La seule influence probable des déformations initiales se limite donc à prédéterminer l'emplacement où se situera la cloque. La pression d'affaissement étant fonction inverse du rayon de courbure, on est en droit de penser que la cloque se produira dans la zone où le rayon de courbure moyen est le plus grand.

L'absence de valeurs polaires ainsi que l'influence très secondaire des déformations initiales oblige à séparer ce phénomène du flambage. C'est pourquoi on devrait utiliser pour le désigner le terme affaissement et non flambage en galerie.

De toute manière, cette théorie n'est établie que pour des blindages lisses, et son extension au cas du raidisseur pose de gros problèmes. En effet, la largeur associée est variable tout au long de la déformée et dépend de l'aspect de cette déformée qui, elle-même, dépend de la variation de la largeur associée. En fait, il semble que ce problème ne puisse être résolu que par itération à l'aide d'un calculateur électronique.

C'est donc avec de nombreuses idées qualitatives, et sans chiffres théoriquement solides que l'essai a été conduit.

\section{III. - CONCEPTION ET REALISATION DE L'ESSAI}

\section{1 - Appareil d'essai.}

Il a été utilisé pour l'essai un appareil conçu et réalisé pour un essai similaire. La longueur entre plateaux de l'appareil $(2,7 \mathrm{~m})$ correspondait à un nombre exact d'intervalles entre raidisseurs du blindage de Roselend. L'appareil est présenté sur la photographie (fig. 2).

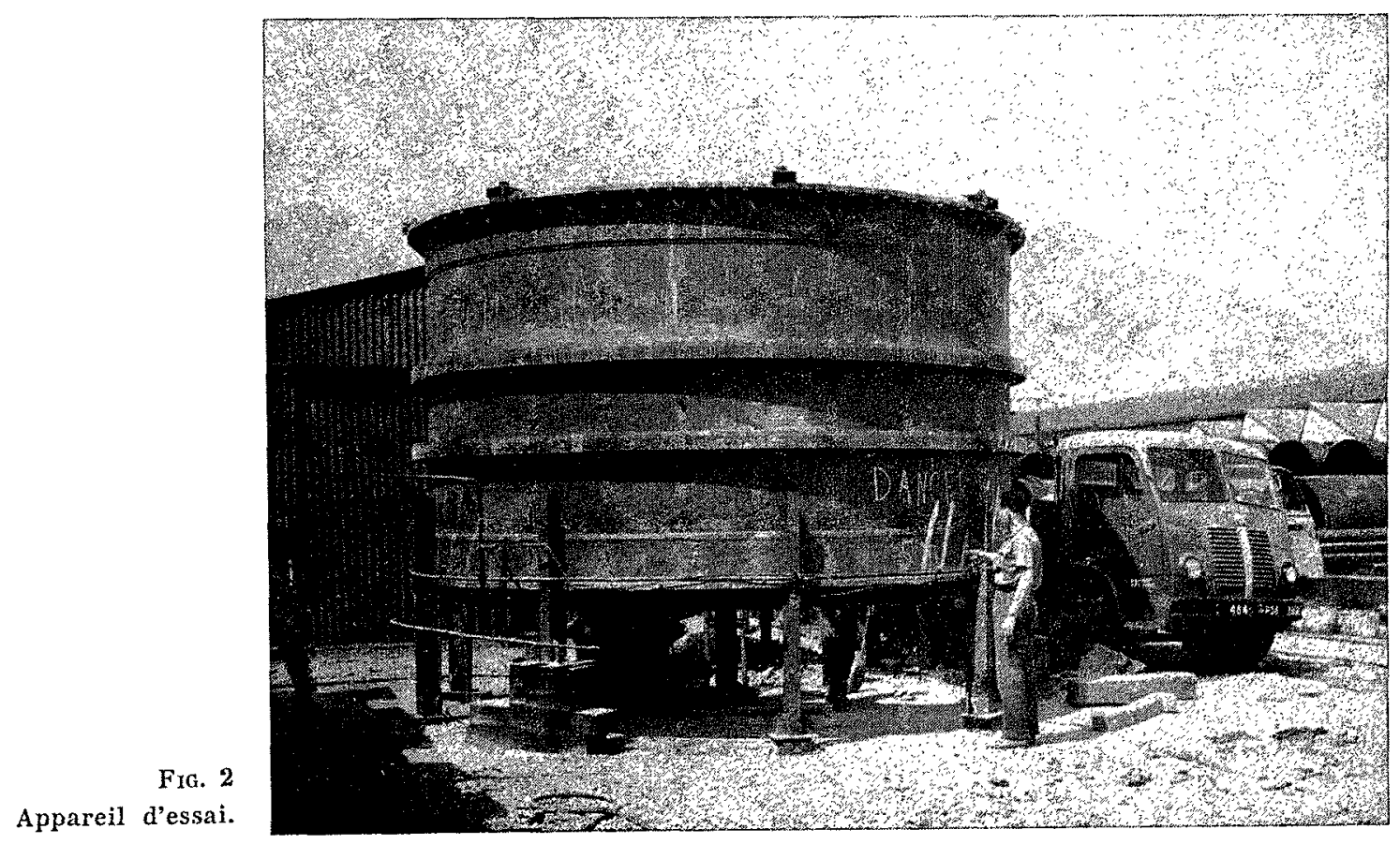




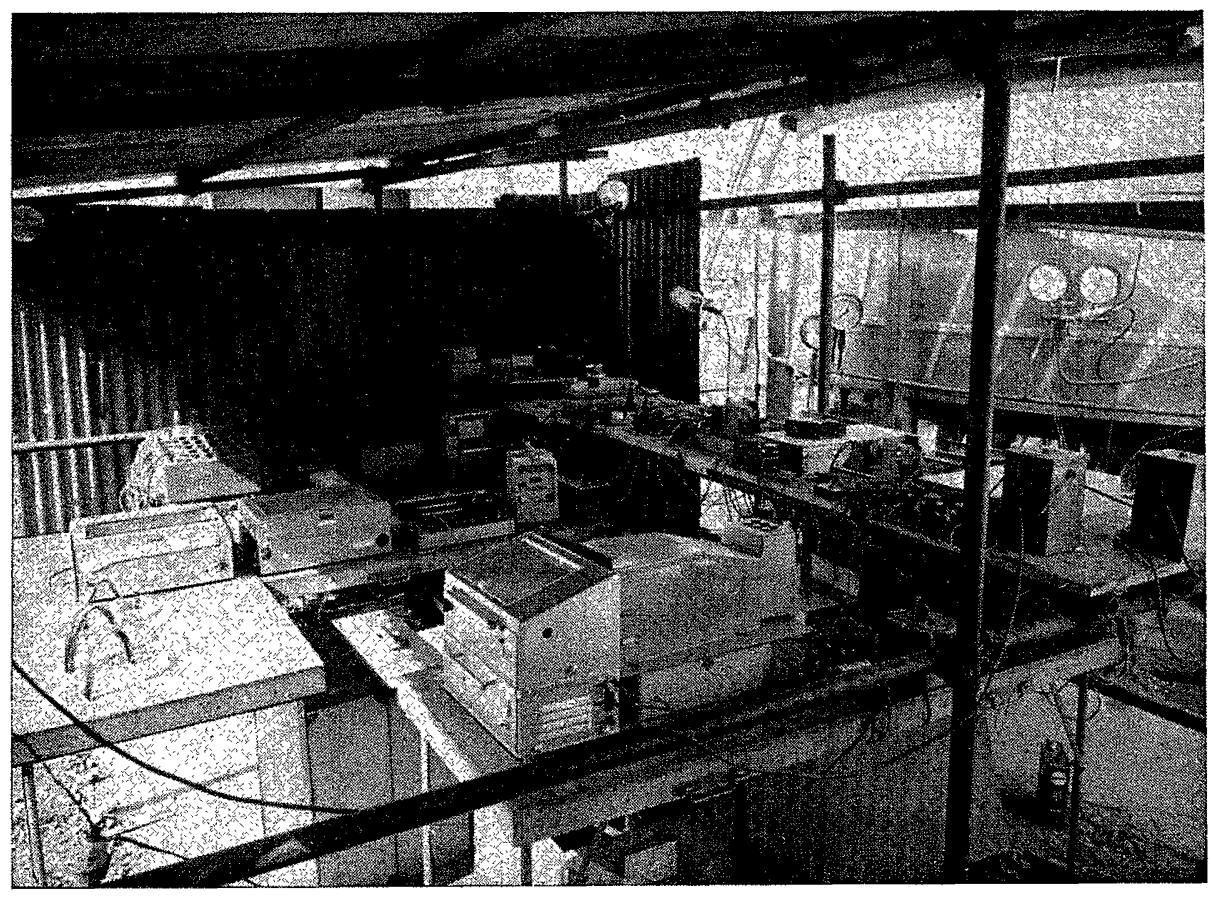

Fig. 3

Poste de commande.
Depuis un poste de commande comportant une pompe et un jeu de vannes by-pass, sortent des tubes de mise en pression; quatre vers l'extérieur du béton, à travers l'appareil lui-même, quatre vers l'intérieur du béton, à travers la virole.

D'autre part, quatre tubulures sortant de l'intérieur, aboutissent au poste de commande et permettent de mesurer la pression effectivement exercée sur la virole. O'n doit noter que la concordance entre les mesures de pression aux divers points a toujours été excellente, tout au long de l'essai.

Les photographies des figures 3 et 4 montrent : - Le poste de commande qui contient, en outre,

Fig. 4

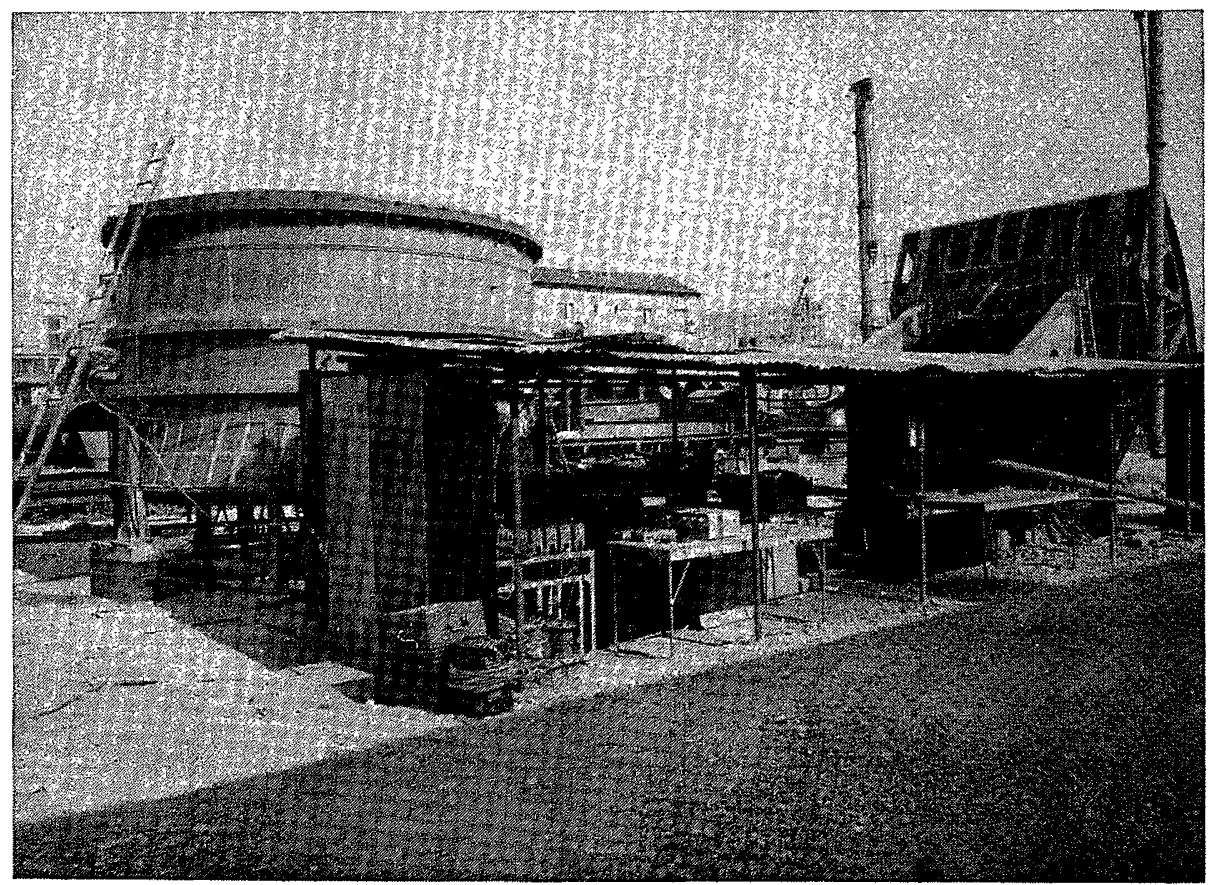

Poste de commande et virole. 


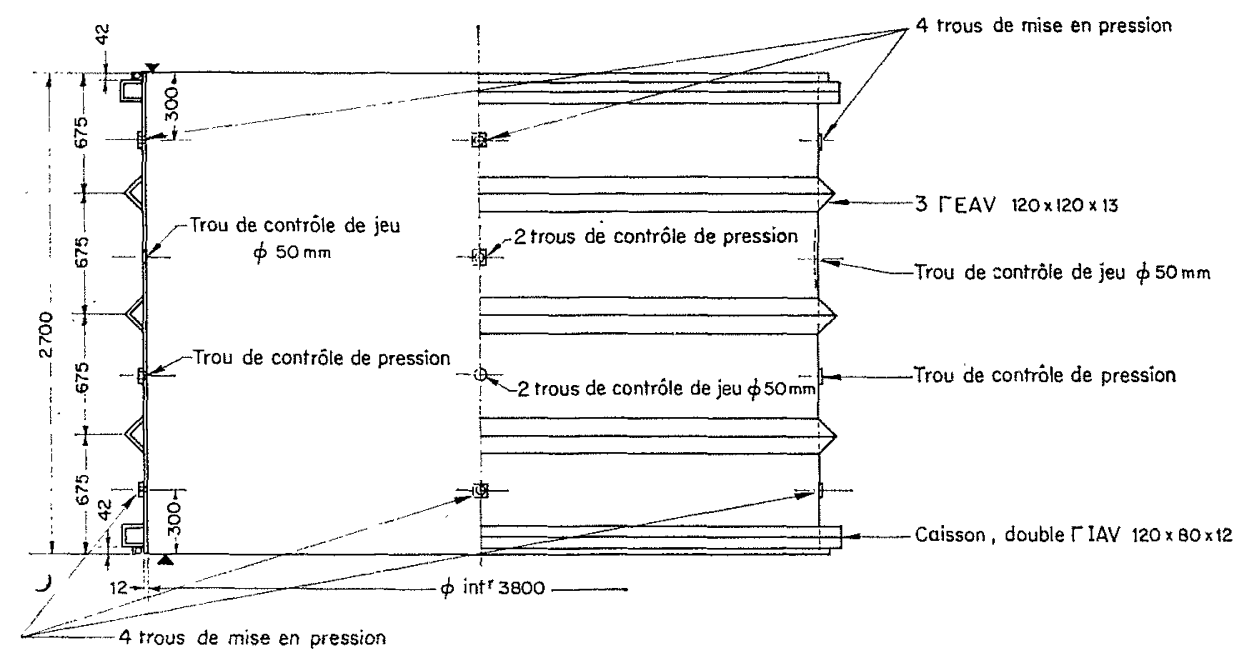

Frg. 5 Virole d'essai.

les appareils nécessaires à la lecture des autres appareils de mesure;

- L'ensemble du poste de commande et de l'appareil d'essai.

\section{III.2 - Virole d'essai.}

La virole d'essai devait ressembler le plus possible à celles utilisées à Roselend. Ceci ne présentait pas de difficultés quant à l'épaisseur, à la section des raidisseurs, à leur espacement, et aux matières utilisées.

Par contre, le problème des extrémités était délicat: un raidisseur important empêcherait les extrémités de suivre un mouvement d'affais-

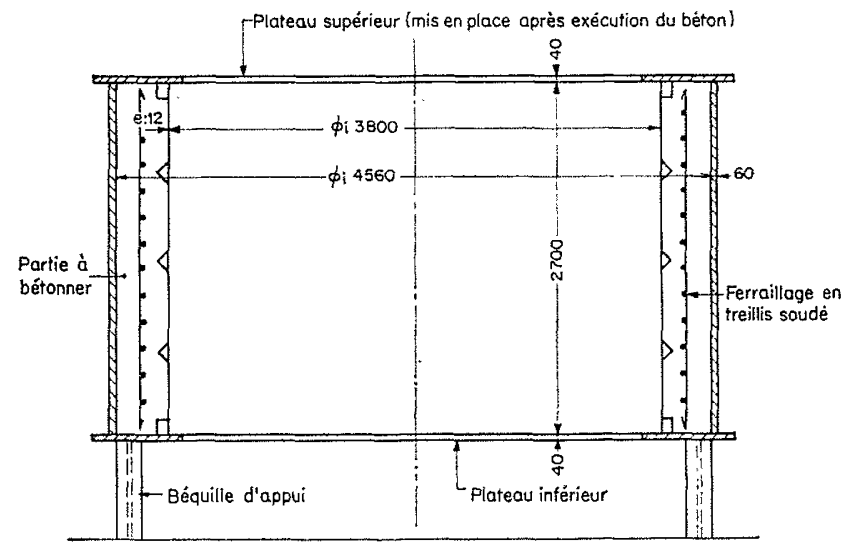

Fig. 6

Essai de résistance aux sous-pressions des blindages de la galerie en charge de Roselend. Schéma du dispositif d'essai. sement du reste de la virole, et un raidisseur faible risquerait de ne pas résister aux torsions créées par la dissymétrie d'extrémité, donc par suite de s'affaisser pour une pression non significative.

Finalement, la disposition des figures 5 et $\mathbf{6}$ a semblé ménager ces divers impératifs : les raidisseurs d'extrémité étant notablement plus importants que les autres, devaient ne pas fléchir, tandis que la présence des deux raidisseurs intermédiaires devait «découpler»suffisamment le raidisseur central des deux extrêmes.

\section{III.3. - Mesures et appareils de mesure.}

Comme il est dit précédemment, il n'y avait pratiquement pas de prévisions chiffrées. Cependant, la pose d'appareils de mesure : jauges de contraintes et jauges de déplacement, pouvait fournir des indications intéressantes concernant :

$1^{\circ}$ L'évaluation de la limite élastique vraie du métal;

$2^{\circ}$ L'allure générale des déformations en fonction de l'espace et du temps;

$3^{\circ}$ La surveillance à distance de l'évolution.

25 paires de jauges de contrainte croisćes et 46 jauges de déplacement avaient été installées.

L'essentiel de ces appareils est visible sur la figure 7 .

Les emplacements de tous ces points de me- 


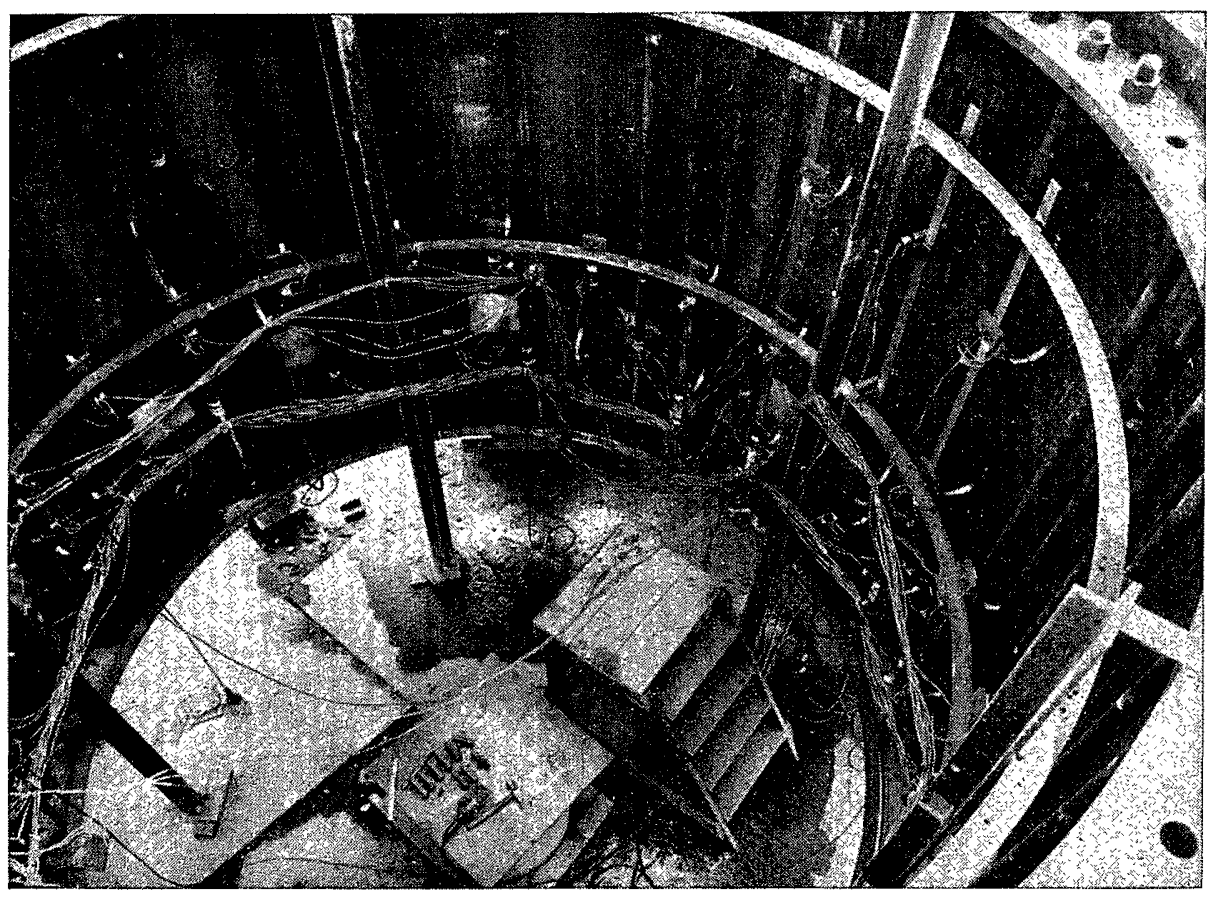

Fig. 7

Essentiel des appareils de mesure.

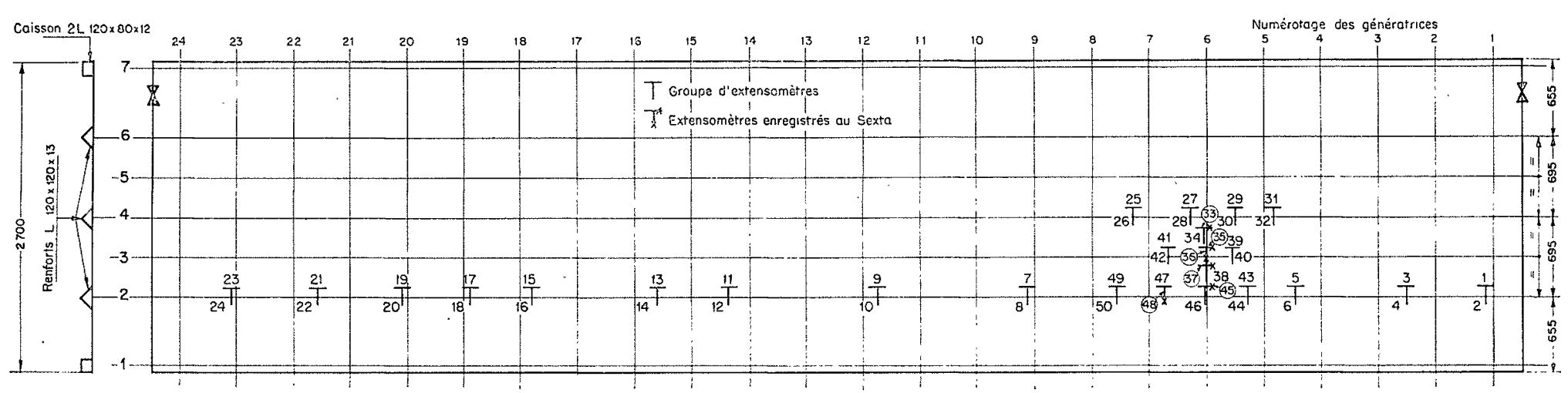

FIG. 8

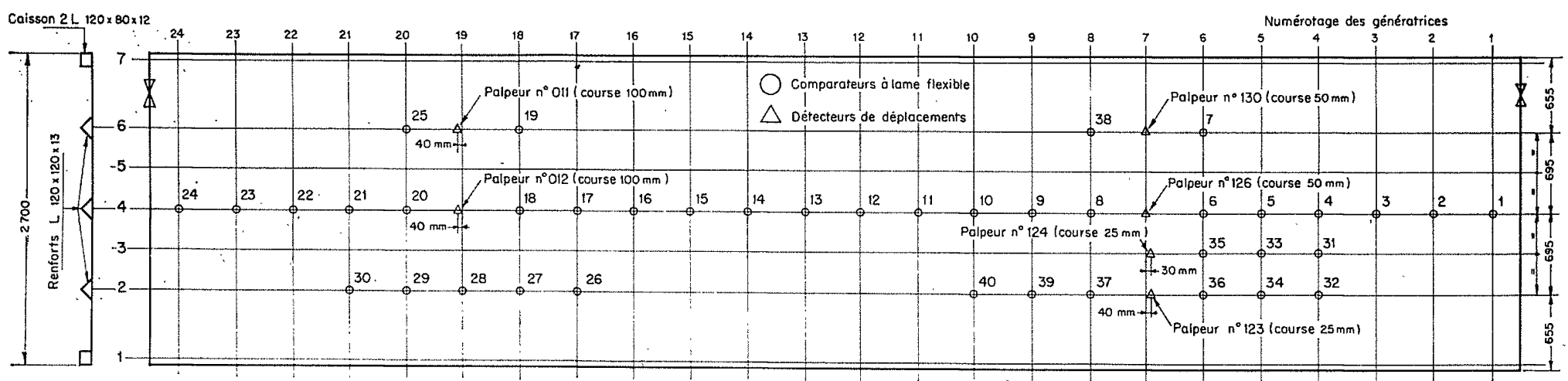

FIG. 9 
Appareil pour la mesure des rayons de courbure.

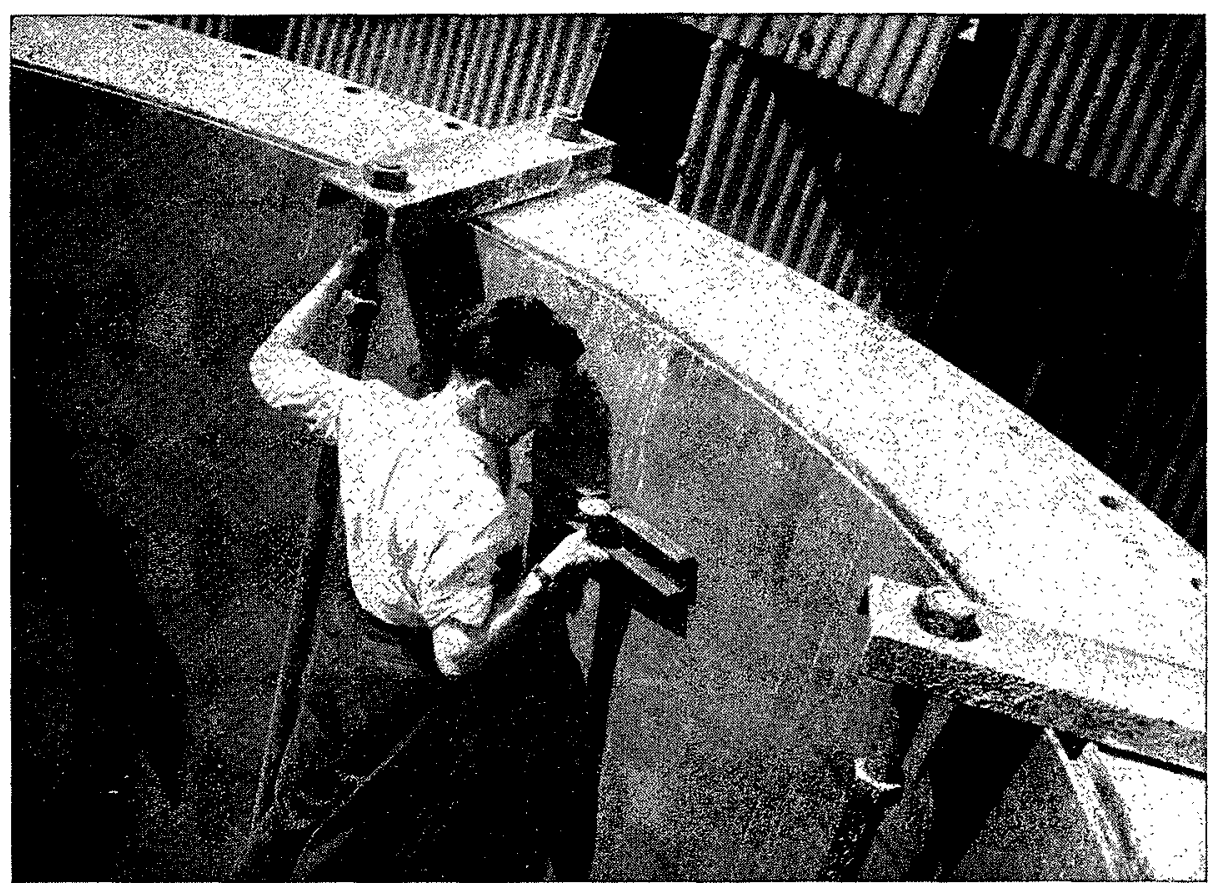

sure sont définis par rapport $\dot{a}$ un développement de la virole sur les figures 8 et 9 .

III.3.1 - MEsures dE DÉFormations inttiales :

Il a été procédé à deux séries de mesures de déformations initiales :

$1^{\circ}$ Mesure des rayons de courbure locale à l'aide d'un appareil à trois pointes. (pointe centrale constituée par un comparateur) de base $400 \mathrm{~mm}$ (fig. 10); $2^{\circ}$ Mesure des rayons polaires à l'aide d'une jauge expansible, par rapport à un axe défini par une visée optique (fig. 11).

\section{$*$}

Chacune de ces mesures a été effectuée pour 168 points répartis à l'intersection de 24 génératrices, régulièrement réparties et numérotées de

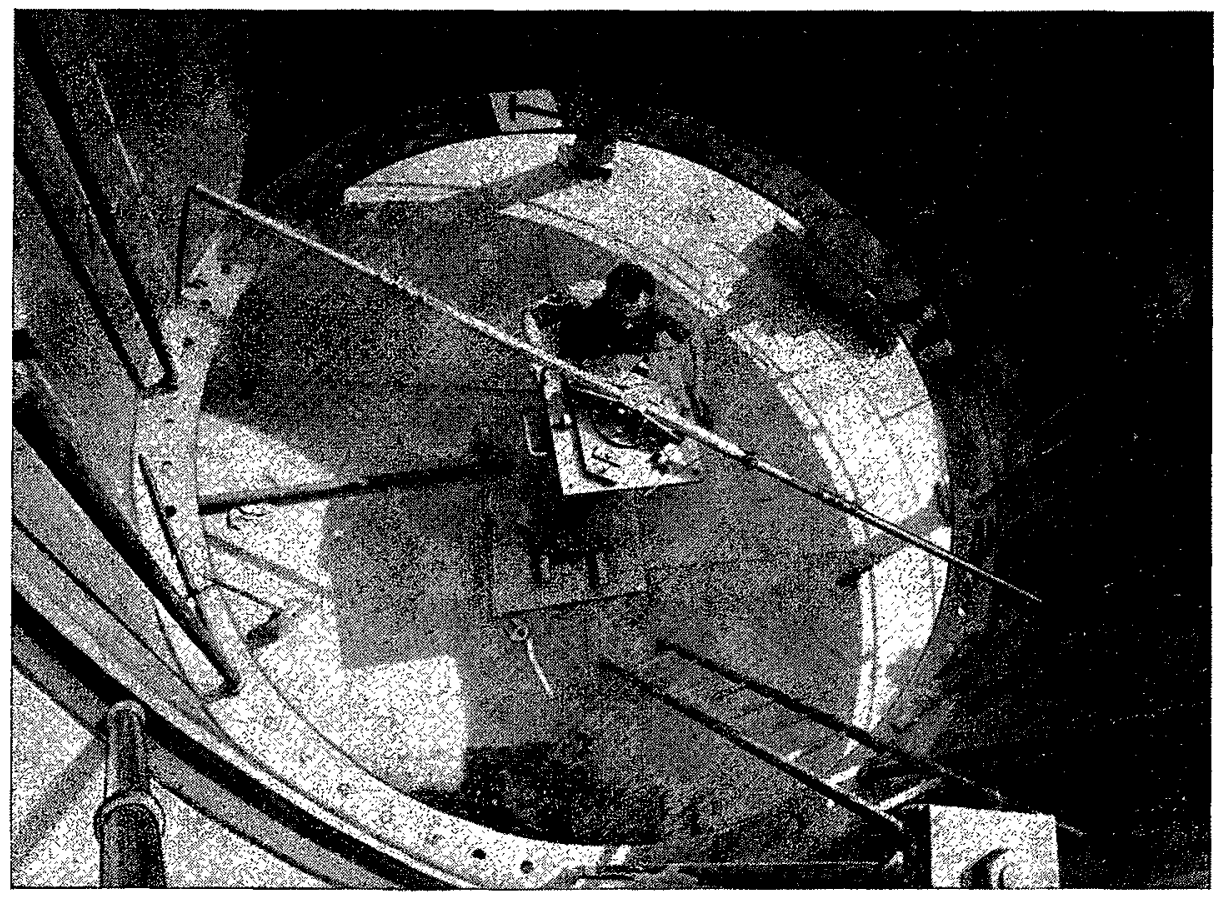

Fig. 11

Jauge expansible pour lat mesure des, rayons polaires. 
1 à 24 à partir de la soudure et de 7 directrices définies par l'axe des cinq raidisseurs et l'équidistance entre le raidisseur central et les deux raidisseurs intermédiaires.

\section{*}

Les buts de ces mesures étaient les suivants :

- Choisir les meilleurs emplacements des appareils de mesure déjà trop peu nombreux pour fournir une vue complète du comportement de la virole;

- Mieux connaître la répartition des déformations initiales d'une virole normalement exécutée, et, en particulier, en connaitre le développement en série de Fourier, très utile pour les études d'affaissement.

Les résultats de ces mesures n'ont vis à vis de l'étude de l'affaissement en galerie, d'autre

\begin{tabular}{|c|c|c|c|}
\hline & \multicolumn{3}{|c|}{ Numéros de génératrices } \\
$\begin{array}{c}\text { Numéro } \\
\text { d'étage }\end{array}$ & 6 & 7 & 19 \\
\cline { 2 - 3 } & & & \\
\cline { 2 - 3 } 2 & 1780 & 2480 & 2060 \\
3 & 1800 & 2130 & 2020 \\
4 & 2000 & 2000 & 2040 \\
5 & 2040 & 2050 & 2020 \\
6 & 2030 & 2030 & 1890 \\
\hline
\end{tabular}

intérêt que de préciser les points de formation préférentielle des cloques; les résultats de mesure et l'analyse harmonique qui en a été faite ne sont donc pas donnés ici dans leur ensemble. Seuls sont indiqués les plus grands rayons de courbure (mesurés sur la base de $400 \mathrm{~mm}$ ).

Tous ces nombres sont donnés en millimètres - la valeur théorique est $1900 \mathrm{~mm}$.

\section{III.4 - Mise en place des appareils de mesure.}

En fonction de ces mesures de déformations initiales, il fut décidé de concentrer les appareils de mesure dans deux zones dont les centres sont définis par les intersections de la génératrice 6 et de la directrice 3 , de la génératrice 19 et de la directrice 4 , comme le montrent nos figures 8 et 9. En effet, si les déformations initiales ne jouent pas en principe un rôle primordial dans le déroulement du phénomène, son début, tant que la virole et le béton ne sont pas encore entrés en contact, est influencé par ces déformations. Elles doivent donc déterminer les points de contact initiaux, et par suite, l'implantation de la ou des cloques, les points les plus vulnérables étant, bien entendu, ceux où le rayon polaire est le plus faible et le rayon de courbure le plus grand.

Afin de contrôler cette hypothèse, un premier essai partiel eut lieu le 3 juillet 1959, qui confirma que la génératrice la plus déformée était bien la génératrice 7 suivie de peu par les génératrices 19 et 20 .

\section{IV. - DEROULEMENT DE L'ESSAI PRINCIPAL}

L'essai principal fut exécuté le 6 juillet. Il comportait des paliers de pression de plus en plus rapprochés, avec la lecture de tous les appareils à chaque paliers.

Les paliers furent les suivants : $10,15,17,5$, $20,21,5,23$ et $25 \mathrm{~kg} / \mathrm{cm}^{2}$.

Jusqu'à $15 \mathrm{~kg} / \mathrm{cm}^{2}$ aucune observation particulière. Les déformations augmentent progressivement, avec prédominance sur les génératrices 19 et 20 (alors que la génératrice 7 pouvait être soupçonnée de préférence). A partir de la pression $20 \mathrm{~kg} / \mathrm{cm}^{2}$ par contre, inversion de la prédominance : c'est la génératrice 7 qui est la plus déformée. Cette déformation n'est d'ailleurs, pas évidente à l'œil : la mesure indique $5 \mathrm{~mm}$ à l'étage 4 et $6,5 \mathrm{~mm}$ à l'étage 2 . Ces déformations sont certainement encore élastiques.

A $21,5 \mathrm{~kg} / \mathrm{cm}^{2}$ apparaissent les premières déformations nettement visibles à l'œil : de nombreuses cloques se forment entre raidisseurs, d'une profondeur de quelques centimètres, à maille nettement carrée.

A chaque formation de cloque, on constate une chute de la pression, puis une remontée lente au fur et à mesure de l'alimentation, puis formation d'une nouvelle cloque... La pression finit par se stabiliser, puis à monter jusqu'à $23 . \mathrm{kg} / \mathrm{cm}^{2}$, au fur et à mesure de la formation de nouvelles cloques.

$\mathrm{La}$ formation des diverses cloques est facilement visible sur l'enregistrement exécuté, où la pression est modulée de petits «tops » toutes les 5 à 10 minutes. Pratiquement, les mesures deviennent impossibles, la pression étant devenue trop instable. On peut, cependant, constater sur place que la déformation de la génératrice 7 
Fig. 12

Vue de la virole après essai.

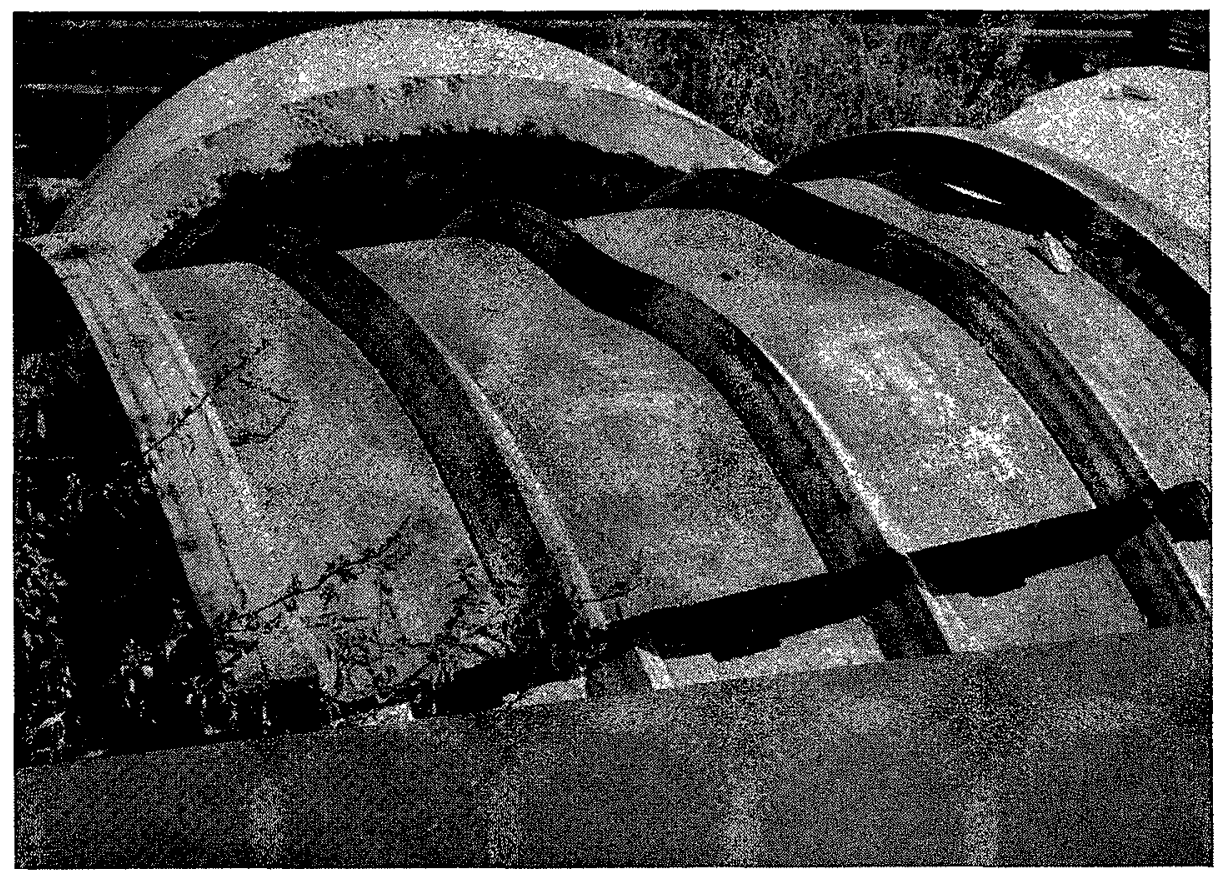

a plutôt diminué au profit de celle des génératrices 19 et 20 (nouvelle inversion).

Les mesures étant devenues impossibles, la pression est «poussée 》 jusqu'au maximum.

Les génératrices 19 et 20 s'enfoncent progressivement, cependant que la génératrice 7 ne progresse plus, puis brutalement se produit à $25 \mathrm{~kg} / \mathrm{cm}^{2}$ une déchirure de la tôle à sa jonction avec le caisson inférieur qui met fin prématurément, semble-t-il, à l'essai.

La virole, et notamment le raidisseur central sont sensiblement déformés et semblent très pro-

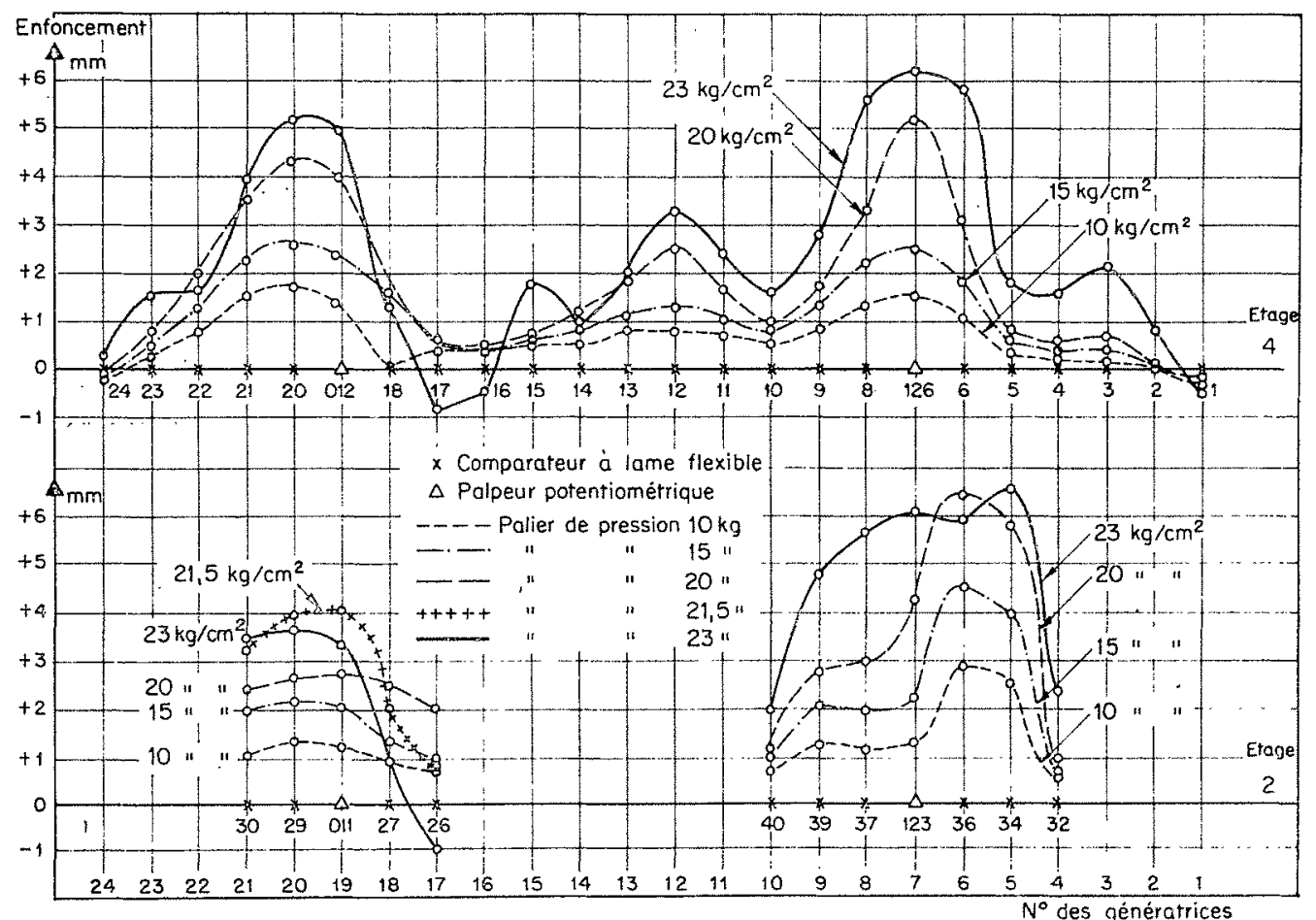

Fig. 13

Blindage Roselend. Déformation des étages 2 et 4 à différents paliers de pression. Essais du 6 juillet 1959. 
ches de la déformation ultime, comme en témoigne la figure 12.

\section{***}

Sur la virole, après démontage, on relève en divers points des traces de glissement sur le béton, longues de deux à trois centimètres. De part et d'autre de la cloque des génératrices 19 et 20 , ces traces sont distantes d'environ 1,5 à $1,6 \mathrm{~m}$, correspondant à un angle au centre d'environ 45 à $50^{\circ}$. Ces traces sont très légèrement perceptibles sur la figure 12.

Enfin, la forme de la cloque correspond assez bien à une fonction de Mathieu, en ce qu'elle présente au centre une courbure bien plus accentuée qu'aux extrémités. Ceci la distingue nettement d'une forme sinusoïdale, dont elle est cependant assez proche dans son ensemble. Il serait hasardeux d'aller plus loin et de comparer exactement son profil avec celui d'une fonction de Mathieu, étant donné ce qui est dit précédemment des «largeurs associées», variables tout au long de la courbe, et ce que l'on peut savoir de l'homogénéité des métaux.

\section{***}

Le relevé des déformations est très suggestif, si l'on fait abstraction de quelques anomalies de la courbe relative à la pression $23 \mathrm{~kg} / \mathrm{cm}^{2}$ (fig. 13) et donne un reflet exact du déroulement du phénomène.

Par contre, les relevés de contraintes sont peu parlants. Malgré la grande densité des appareils de mesure, les allongements relevés ne varient pas d'une manière suffisamment continue entre les diverses jauges.

\author{
Résultats de l'essai; \\ comparaison aux résultats de calcul
}

Si l'on compare les résultats à ceux des carculs qui donnent :

- Pression de flambage de la tôle entre raidisseurs : $37 \mathrm{~kg} / \mathrm{cm}^{2}$ (contre 21,5 à l'essái);

- Pression d'affaissement du raidisseur : $24 \mathrm{~kg} / \mathrm{cm}^{2}$ (contre 25 à l'essai);

on constate :

$1^{\circ}$ Une grosse divergence en ce qui concerne la pression de flambage entre raidisseurs. Cette divergence s'explique facilement, car il a fallu, pour choisir le coefficient $K$ de Sturm, prolonger une courbe de l'abaque dans une zone où ces coefficients varient très rapidement. Il serait préférable dans un tel cas, de recalculer selon la théorie le coefficient de Sturm, dans la bande considérée. Une bonne approximation pourrait être obtenue à l'aide de la théorie du flambage des voiles plans; en effet, les cloques sont très courtes devant le développement de la virole, et l'influence de la courbure est par conséquent faible;

$2^{\circ}$ Une très faible divergence, en ce qui concerne l'affaissement du raidisseur. Ce fait peut paraître plus difficile à interpréter, étant donné que la théorie de E. Amstütz, qui a servi aux calculs, semble mal fondée mathématiquement. Cependant, on observe que la déformée choisie par E. Amstütz n'étant pas très éloignée de la déformée théorique, et la largeur associẻe choisie, bien qu'approximative, étant également de l'ordre de grandeur de la largeur associée réelle, il était normal que le résultat obtenu soit proche de la réalité.

\section{CONCLUSION}

De ces diverses observations, on peut conclure :

- Que moyennant la modification simple du procédé de calcul du flambage entre raidisseurs, la méthode de dimensionnement actuellement employée par les Etablissements Bouchayer et Viallet est valable;

- Que la théorie de M. Simon-Suisse est justifiée :

a) par sa bonne concordance avec les résultats sur viroles lisses;

b) par la concordance avec la déformée observée lors de l'essai relaté dans la présente communication; c) par le fait que des impacts très nets et bien localisés aux extrémités de la cloque ont été observés;

d) par le fait qu'il s'est formé deux cloques au début de la montée en pression, mais que l'oscillation de leurs amplitudes relatives et la formation d'une seule cloque finale ont bien prouvé cette tendance à n'en former qu'une.

$$
\text { 获 }
$$

Tous les éléments théoriques sont réunis pour exécuter l'extension de la théorie de M. SimonSuisse au cas des viroles renforcées : théorie du flambage en galerie des viroles lisses, et théorie des largeurs associées pour les formes de révolution. 